\title{
Significados de la corrupción en universitarios dominicanos, docentes en formación
}

\author{
Meanings of Corruption in Dominican University Students, \\ Teachers in Training
}

\author{
Fernando García-Leguizamón ${ }^{\text {A }}$ ORCID: 0000-0003-4796-7799 \\ Recibido: 26/7/2019・Aprobado: 2/8/2019
}

\begin{abstract}
Cómo citar: García-Leguizamón, F. (2019). Significados de la corrupción en universitarios dominicanos, docentes en formación. Ciencia y Educación, 3(2), 19-35. Doi: https://doi.org/10.22206/cyed.2019.v3i2.pp19-35
\end{abstract}

Resumen

De manera general, la corrupción se caracteriza como el abuso de un poder encargado, con el fin de obtener un beneficio particular. La gran corrupción ha sido abundantemente abordada desde diferentes perspectivas en las ciencias sociales, pero solo en tiempos relativamente recientes se ha otorgado una mayor importancia al estudio de la pequeña corrupción, la que emerge y se reproduce en prácticas cotidianas que se confunden con la lógica del intercambio privado de favores. Este interés ha implicado la adopción de una mirada culturalista para la comprensión de los significados que en contextos particulares le asignan los sujetos al fenómeno. Siguiendo este camino, nos preguntamos aquí por los significados que un grupo de estudiantes universitarios articulan colectivamente en torno a la corrupción, cuando se encuentran en situaciones sociales de habla. Suponemos que las interacciones lingüísticas que allí ocurren permiten identificar repertorios interpretativos, esto es, recursos culturales compartidos, disponibles para la descripción y la evaluación del fenómeno, recursos con los que los sujetos adoptan frente a la corrupción una posición no exenta de variaciones y tensiones. El acercamiento a estos significados se realizó desde una perspectiva cualitativa, empleando la técnica
In general terms, corruption has been characterized as the abuse of a commissioned power, in order to obtain a particular benefit. Grand corruption has been abundantly approached from different perspectives in the social sciences, but only in relatively recent times has greater importance been given to the study of "petty" corruption, which emerges in practices of everyday life mingled with the logic of private exchange of favors. This concern has implied the adoption of a culturalist viewpoint for the understanding of the meanings that people assign to the phenomenon in particular contexts. Following this path, we want to explore the meanings that a group of university students in social speech situations collectively articulate around corruption. We assume that the linguistic interactions that occur there allow us to identify interpretive repertoires, that is, shared cultural resources, available for the description and evaluation of the phenomenon, resources through which people take a stance against corruption, that is not free of of variations and tensions. The approach to these meanings was made from a qualitative perspective, using the technique of focus group. The population of the study are university students in the career of Education, in the city of San Pedro de Macorís, in the Dominican Republic. Three interpretative

A. Docente del Instituto Superior de Formación Docente Salomé Ureña, Recinto Juan Vicente Moscoso, San Pedro de Macorís. Correo-e: fernando.garcia@isfodosu.edu.do,garcía.leguizamon@gmail.com 
del grupo focal. La población del estudio son estudiantes de la carrera de educación, en la ciudad de San Pedro de Macorís, en República Dominicana. Identificamos tres de estos repertorios: el de la victimización, el de la aceptación-justificación y el de la superación ética.

Palabras clave: corrupción; formación de docentes; estudiante universitario; análisis del discurso, hermenéutica.

\section{Introducción}

La formación de ciudadanos que comprendan e interioricen los principios fundamentales de la vida democrática es parte esencial de la misión de nuestros sistemas educativos. Acorde con esto, no puede sernos indiferente una problemática que se levanta como obstáculo para la construcción de una ética pública: la corrupción. Emprender acciones pedagógicas frente a ella es una tarea que demanda entender sus causas estructurales, pero también la manera como se viviencia, se evalúa y se reproduce sutilmente en la vida cotidiana.

Desde la segunda mitad del siglo xx, tanto en la sociología como en la ciencia política, se han propuesto significativas miradas macro sobre la corrupción, pero encontramos que los abordajes antropológicos emprendidos más recientemente ofrecen una perspectiva más rica, quizá, para los propósitos educativos, en cuanto permiten enfocar las imágenes culturales que le dan sentido a la corrupción en micro escenarios cotidianos, susceptibles de ser objeto de acciones educativas transformadoras.

El presente estudio explora los significados que tiene la corrupción para un grupo de jóvenes, estudiantes universitarios, que se preparan para ser docentes escolares.

Entendemos esta exploración como una contribución para el diseño de estrategias pedagógicas específicas dentro de la formación ética y ciudadana.

\section{Referentes teóricos}

En su sentido básico, se entiende por corrupción el abuso de un poder encomendado con el propósito de obtener beneficios particulares. La preocupación repertoires were identified: victimization, acceptance-justification, and ethical overcoming.

Keywords: Corruption; teacher training; college student; discourse analysis, hermeneutics.

de los estudiosos del fenómeno en las ciencias sociales se ha enfocado en la corrupción política, se ha puesto el énfasis en el abuso de los cargos públicos, considerándose que se presenta corrupción "dondequiera que el tenedor de un cargo público, mediante recompensas monetarias o de otro tipo, es inducido a realizar acciones que favorecen a quien provee la recompensa, perjudicando por tanto al grupo u organización a la que dicho funcionario pertenece." (Friedrich, 2002, p. 15).

Aunque no se trata de una problemática exclusiva de los países en vía de desarrollo, sí se constata en estos una mayor presencia de corrupción (Transparencia Internacional, 2018). De allí que se asocie con su origen el bajo ingreso de los países, la limitación en la apertura de sus economías, la influencia de la religión (la corrupción es menor en países protestantes), la reducida libertad de los medios de comunicación y los bajos niveles educativos (Svensson, 2015). Puesto que no es posible establecer una relación monocausal en su origen, se asume que en ella convergen diferentes factores: el entorno político y económico, la ética profesional, la legislación y los elementos culturales (Šumah, 2018).

En el abordaje teórico de la corrupción se distinguen tres grandes perspectivas, según se ponga el acento en los cargos públicos, en la lógica del mercado, o en el interés público (Heidenheimer, y Johnston 2002; Kajsiu, 2015). En el primer sentido, la corrupción se asocia al abuso de autoridad para un beneficio personal, y se describe como:

el comportamiento que se aparta de los deberes normales de un rol público en razón de consideraciones privadas (familia, círculo social cercano), ganancias pecuniarias o de estatus. Esto incluye conductas como soborno, (uso de recompensa para viciar el juicio de una persona 
en una posición de confianza), nepotismo (asignación de patrocinios por motivo de afiliación personal, antes que por mérito), y malversación (apropiación ilegal de recursos públicos para usos privados). (Nye, 2002, p. 284)

En esta perspectiva se parte del ideal de modernización, basado en el modelo weberiano de dominación legal racional, en el que la conducta de los funcionarios se ajusta a las reglas formales de un rol, claramente diferenciadas de las que guían su vida privada, de lo que deriva un trato imparcial a los clientes.

A mediados del siglo $\mathrm{xx}$, este enfoque permitía interpretar los fenómenos de abuso de poder, soborno, clientelismo, nepotismo y peculado que emergían en los países tercermundistas como expresión de una modernización "incompleta”, allí donde no se había logrado consolidar una administración libre del personalismo y el autoritarismo de las sociedades tradicionales, lo que se convertía en obstáculo para la adopción de prácticas de eficiencia, imparcialidad y meritocracia (Kajsiu, 2015). Sin embargo, al menos desde el último tercio de siglo, se puede establecer que la corrupción no es un fenómeno exclusivo de un tercer mundo "desviado" frente a un primer mundo "normal", sino que se presenta también en los países desarrollados, relativizándose de esta forma los supuestos del paradigma de la modernización, que compite ahora con un enfoque del problema que echa mano de las herramientas de la teoría económica y sus supuestos comportamentales: incluso los funcionarios son egoístas racionales que buscan maximizar su beneficio.

En este segundo tipo de aproximación a la corrupción (Heidenheimer y Johnston, 2002; Kajsiu, 2015), el abuso del cargo para fines privados, predominantemente a través del soborno, sería una forma de maximización del ingreso por parte del funcionario y las rentas, así aseguradas, representarían "retornos" por la posición monopolística que generan restricciones introducidas por el gobierno (por ejemplo, adjudicación de licencias). El funcionario, como cualquier actor en el mercado, sopesa los beneficios del soborno frente a los costos que conlleva ser atrapado. Klitgaard (1988), citado por Kajsiu (2015), plasma esta idea en una sencilla fórmula: corrupción es igual al monopolio de poder, más la discrecionalidad, menos la accountability u obligación de rendir cuentas $(\mathrm{C}=\mathrm{M}+\mathrm{D}-\mathrm{A})$. En otras palabras, la corrupción es traducible a la oportunidad de ser corrupto, cuando los beneficios superan los posibles costos.

La tercera perspectiva de abordaje de la corrupción que presentan los autores mencionados, se pregunta por sus efectos para la democracia, ya sea que esta se conciba en un sentido normativo fuerte como un sistema en el que los ciudadanos deben tener la posibilidad de participar en el procedimiento para el logro de acuerdos y la toma de decisiones que los afectan, o simplemente se describa como un sistema que satisface las condiciones de que "el gobierno esté sometido al rendimiento de cuentas a los ciudadanos, que sus administradores legislen de manera equitativa, que sus acciones sean transparentes, y que todos los ciudadanos tengan un igual acceso al proceso político" (Anderson y Tverdova, 2003, p. 91, citado por Kajsiu, 2015 , p. 27). No solo en el primer caso es claro que la corrupción produce exclusión e injusticia en cuanto introduce distinciones a partir del poder económico, sino que el incumplimiento de las condiciones, en el segundo, permite constatar empíricamente que donde hay corrupción los ciudadanos reportan menores niveles de satisfacción con los rendimientos de su sistema político, lo perciben como menos legítimo y profesan menor apoyo a sus instituciones (Seligson, 2002).

Dobovsek (2012, citado por Šumah, 2018) sostiene que la corrupción es menos un problema de debilidad de las personas que de las instituciones, que no logran fijar límites a la codicia y las tentaciones individuales. Habría, sin embargo, que precisar que las instituciones no son solo legales y formales, sino también morales e informales, lo que nos remite al ámbito de la cultura. En ese contexto, análisis recientes han redirigido la mirada desde el terreno del Estado como único culpable de la corrupción, para enfocar su presencia y reproducción dentro de la sociedad a través de instituciones informales cimentadas en redes que se extienden por el aparato estatal y atraviesan las fronteras entre Estado y sociedad (Stefes, 2007), donde se integran elementos de diferentes órdenes normativos y simbólicos, a partir de los que la corrupción adquiere significados que se distancian de los que asumen quienes tradicionalmente 
la explican y buscan combatirla desde visiones teóricas homogenizantes (Torsello, 2011; Huber, 2008; Zalpa, Tapia y Reyes, 2014).

Las perspectivas sociológica, economicista y filosófico-política de la corrupción la sitúan predominantemente en el ámbito del Estado y ofrecen miradas macro que evalúan la corrupción desde supuestos formales, pero no alcanzan a enfocar los sentidos que de ella construyen las personas en interacciones cotidianas, lo que permite entender por qué en los últimos años han ganado importancia los acercamientos culturalistas al fenómeno. De esta manera se ha documentado, por ejemplo, cómo el compadrazgo en México, el guanxi en China y el blat en Rusia son prácticas en las que se traslapan las lógicas del regalo y el soborno, de manera que "lo que las autoridades consideran corrupto e ilegal, la población local lo considera como donación legítima de obsequios" (Graycar y Jancsics, 2016, p. 3). Ambas prácticas, el regalo y el soborno, comparten una característica: son comportamientos sociales que producen reciprocidad y estabilidad, llenando los vacíos que dejan las instituciones formales y reduciendo la aspereza en ambientes de incertidumbre. El compadrazgo en América Latina ha permitido la supervivencia física a grupos sociales pobres. El blat en la Rusia comunista, como forma de trueque, reducía la incertidumbre en situaciones de escasez. El guanxi en China construye confianza en medio de una tensa burocracia y en ausencia de instituciones legales o comerciales funcionales.

Algo similar constata un estudio en El Alto, Bolivia: "los políticos están facultados, y se espera que así lo hagan, para acumular riqueza personal y usarla en beneficio de su red social, que incluye inicialmente a toda su familia" (Lazar, 2005, p. 223, citado por Gephart, 2012, p. 5) Narrativas de una aparente aceptación de la corrupción se encuentran también en Benin, Nigeria y Senegal, donde desviar las reglas se considera un acto de cortesía: "En el contexto local se le llama a alguien corrupto, no cuando rompe la ley en beneficio personal, sino cuando la ganancia personal resultante de la práctica ilegal se percibe como exagerada" (Gephart, 2012, p. 6). Más allá de las acotadas definiciones de los científicos sociales, señala Gephart, parece que "la definición que los actores elijan depende de asuntos prácticos y de la manera como conceptualicen el mundo social y su posición en él" (p. 8).

Ahora bien, si la cultura es "el significado social de la realidad" (Zalpa, et al., p. 173), los acercamientos culturalistas a la corrupción podrían sugerir que diferentes grupos humanos ordenan sus prácticas a partir de significados diferentes, de manera que no existiría una noción universal de corrupción, por fuera del ideal liberal occidental que suelen tener los analistas. Saliendo al paso a este relativismo, Rothstein y Torsello (2013) han mostrado que no existe geográfica e históricamente una comprensión diferente de la corrupción basada en diferentes comprensiones morales, sino que las diferencias percibidas responden más bien a variaciones en la magnitud y el tipo de lo que en las distintas sociedades se considera como "bienes públicos". Independientemente de su nivel de desarrollo, todas las sociedades tienen que producir algún conjunto mínimo de bienes públicos: una organización colectiva para su seguridad física, el abastecimiento colectivo de agua y comida, el apoyo organizado para los huérfanos, etc. Cuando aquellos que son responsables por el manejo de tales bienes públicos los convierten en bienes privados, las personas, por lo general, consideran sus actos como moralmente incorrectos.

Según Rothstein y Torsello (2013), esta comprensión universal de la corrupción permitiría entender la valoración negativa que empíricamente se constata en diferentes culturas frente a cierto tipo de acciones (preferencias de un funcionario por sus amigos para la distribución de recursos; de familiares para la asignación de empleos sin la debida cualificación; o la exigencia de pagos adicionales para realizar servicios incluidos ya en sus funciones), a las que, antes que "incorrectas, pero comprensibles", se les considera "incorrectas y sancionables". Tal valoración no implica, sin embargo, que se dejen de cometer y, eventualmente, de justificar tales actos, ya que las situaciones en las que tienen lugar se le presentan al actor como un problema de "acción colectiva", donde resulta poco razonable, por ejemplo, abstenerse de ofrecer o recibir sobornos o recurrir a padrinos, cuando se trata de prácticas generalizadas que determinan las expectativas de comportamiento en su grupo social, con altos costos para quien se abstiene de realizarlas. 
Al abordar la corrupción poniendo de relieve los sentidos compartidos que acompañan implícitamente las acciones e interacciones cotidianas de los sujetos, relegamos a un segundo plano el interés por su percepción de los escándalos que resuenan mediáticamente desde el centro del sistema político y administrativo. La atención se dirige a la forma como el fenómeno se vive y se reproduce, a veces de manera desapercibida y naturalizada, en el contacto cotidiano entre el pequeño funcionario y el ciudadano, donde se transa la apropiación o usufructo privado de pequeños bienes públicos. En este sentido, es útil mencionar la distinción formulada por Aroca (s.f., citado por Zalpa, et. al, 2014), entre corrupción negra, gris y blanca. La negra corresponde a la gran corrupción política (caso Odebrecht), que los organismos internacionales y la población coinciden en definir como tal. La gris se asocia con acciones que solo algunos miembros de la sociedad (académicos, periodistas, etc.) consideran como corrupción, pero la mayoría no ("mordidas", pequeños pagos de dinero a funcionarios de menor nivel, que se disfrazan como propinas). La blanca, finalmente, es aquella que los organismos internacionales definen como corrupción, pero los miembros de la sociedad no la perciben como tal (intercambio de favores por relaciones de amistad, lazos familiares o redes de influencias).

Como observa Huber (2008), los actos de corrupción son usualmente clandestinos y se resisten a la observación participante, de manera que para acercarnos a ella "dependemos de observaciones informales y discursos cotidianos de la gente, que son ricas en anécdotas, confesiones y acusaciones, y a través de las cuales se puede obtener confirmaciones y valoraciones sobre prácticas corruptas" (Sissener 2001, citado por Huber, 2008, p. 5). Además, puntualiza este autor, puesto que se trata de un concepto cargado de connotaciones ético-morales negativas, es inevitable un sesgo en la recopilación de los datos, del que resulta que los corruptos siempre son los "otros". Estas observaciones, que anticiparían un obstáculo para la aproximación a los "hechos duros" cuando se pretende estudiar el fenómeno de la corrupción, en nuestro caso confirman más bien la disponibilidad de un amplio objeto de análisis: los discursos sobre la corrupción. Para nuestros propósitos, no tenemos que suponer que tales discursos sean el reflejo más o menos fiel de una realidad objetiva externa, ni la proyección de estados mentales de los sujetos. Nos interesa básicamente su emergencia en interacciones en las que los individuos hacen uso de recursos culturales compartidos, seleccionando en ellos lo que les permite dotar de sentido a un fenómeno como la corrupción.

Estos recursos compartidos son repertorios interpretativos, que Wetherell y Potter (1996) describen como medios empleados por los hablantes para construir versiones de sus acciones, procesos cognitivos y fenómenos sociales. Dichos repertorios están constituidos por un conjunto de términos usados de un modo específico y derivan por lo general de una o más metáforas centrales. Los discursos organizados en repertorios no representan una inflexible imposición sobre el individuo, sino que son recursos disponibles socialmente frente a las que el sujeto tiene la posibilidad de posicionarse dinámicamente, de manera que se reconoce "la agencia humana dentro del despliegue flexible del lenguaje": los repertorios disponibles "permiten una comprensión compartida y son como los pasos prefigurados de un baile, que pueden ser unidos de manera flexible y creativa en cada nueva performance" (Edley, 2001, p. 198).

A propósito de la alternancia en las posturas de reprobación y justificación de actos de corrupción que algunas veces parecen exhibir los sujetos, la teoría de los repertorios interpretativos permite concebir que en un discurso se puedan presentar valoraciones opuestas, sin que ello conduzca necesariamente a su exclusión recíproca. Por una especie de "conmutación" (switching, Duit, 2008), distintos repertorios pueden emplearse en diferentes momentos, de manera que la acción cuestionable en uno de ellos puede resultar justificada dentro de otro. De este modo, además de poner énfasis en las funciones del lenguaje (explicar, justificar, etc.) y en su capacidad de realizar acciones, este enfoque otorga importancia a la variabilidad lingüística de los hablantes, a la emergencia de perspectivas y evaluaciones cambiantes y divergentes de los fenómenos, acciones y procesos que tienen lugar en el mundo social. En esta perspectiva analítica importa menos evaluar la coherencia de una posición ideológica definida que resaltar la diversidad y la 
multiplicidad explicativas de un fenómeno y las justificaciones según la situación.

Como señalan Wetherell y Potter (1996), la variación no ocurre de manera deliberada o intencional, pues los sujetos no actúan planeando y ajustando estratégicamente sus discursos, sino que más bien dicen lo que les parece adecuado a la situación. Esto no implica la simple adopción de una estructura preestablecida que excluya interpretaciones alternativas, sino que los repertorios aparecen como un recurso en cuya inteligibilidad compartida se apoya el hablante, para luego actualizar una posición, produciendo una definición de sí que puede reflejar una tensión entre repertorios divergentes.

\section{Metodología}

Empleamos la técnica del grupo focal para producir discursos sobre la corrupción, dentro de ellos los repertorios interpretativos son nuestra unidad de análisis. La técnica adoptada propicia una situación de interacción entre los participantes y permite obtener información sobre los significados que subyacen a las apreciaciones grupales, arrojando luz sobre las comprensiones normativas en las que se apoyan sus juicios colectivos (Bloor, Frankland, Thomas y Robson, 2001). El grupo focal ofrece un escenario en el que se explicitan y examinan supuestos normativos que usualmente se asumen como correctos y compartidos y cuya fuerza radica precisamente en que se dan por sentados. La evaluación que se abre, en ese sentido, permite a su vez identificar ambigüedades y contingencias que rodean los órdenes normativos cuando ellos se exponen desde las excepciones que tienen lugar en las prácticas cotidianas.

La población de este estudio son estudiantes dominicanos del Instituto Superior de Formación Docente Salomé Ureña (ISFODOSU) en su sede de San Pedro de Macorís. Se realizaron tres grupos focales en el segundo semestre del 2018, con una duración aproximada de hora y media cada uno, conformados por estudiantes de tres cuatrimestres diferentes, en la carrera de Lenguaje y Ciencias Sociales en Educación Básica. Como criterio inicial para la escogencia de los grupos se tuvo en cuenta que no existiera todavía un conocimiento personal entre los estudiantes y el docente investigador en ninguna de las asignaturas que imparte. En segundo lugar, se optó por grupos que no hubieran cursado aún la asignatura de "Ética y ciudadanía", en la que se tocan temas como la corrupción y el desarrollo del juicio moral, lo que posiblemente hubiera condicionado el contenido de las intervenciones.

En tercer lugar, puesto que se trataba de nuestro primer acercamiento empírico a un fenómeno frente al cual no podíamos todavía señalar posibles posturas para darles una representación en las discusiones, se consideró adecuado adoptar para la muestra un criterio de conveniencia, a través de la invitación a tomar parte voluntariamente en lo que se describió a propósito, y con cierta vaguedad, como una conversación sobre la vivencia que jóvenes estudiantes tenían de algunos aspectos de su propia cultura. Puesto que el moderador era extranjero, pero hispanoparlante, la solicitud se recibió con naturalidad y se respondió con una actitud de colaboración.

Finalmente, buscamos que en los grupos se diera un equilbrio en la representación de sexos. Numerados en el orden cronológico de su realización, el grupo 1 estuvo conformado por siete estudiantes de séptimo cuatrimestre: cuatro mujeres y tres hombres, con edades entre los 20 y los 23 años. En el grupo 2 participaron ocho estudiantes de primer cuatrimestre: cinco hombres y tres mujeres, con edades entre los 16 y los 23 años. Al grupo 3 asistieron ocho estudiantes de sexto cuatrimestre: cuatro mujeres y cuatro hombres, con edades entre los 18 y los 20 años.

En los encuentros no se mencionó inicialmente la palabra "corrupción" para evitar una activación temprana de las valoraciones que normalmente se expresan en espacios formales. Siguiendo la estrategia de Zalpa, Tapia y Reyes (2014), se adoptó como estrategia inicial de focalización la interpretación de algunos refranes populares ("evangelios chiquitos"), en tanto ellos condensan comprensiones compartidas y regulaciones sociales tácitas. Esto permitió la tematización de la importancia de los vínculos sociales y el intercambio de favores, desde lo cual los participantes derivaron espontáneamente a considerar situaciones ambiguas que introdujeron la pregunta por la corrupción. 
Activada ya la dinámica propia de discusión, se buscó en un segundo momento que hicieran explícito su posicionamiento frente a prácticas cotidianas de asignación de recursos públicos, lo que asociaron con situaciones de su propio contexto y con anécdotas y creencias sobre el nombramiento magisterial. Finalmente, tratamos de visualizar la comprensión que tenían los participantes de su ubicación en el espacio social, en los límites y relaciones entre lo público y lo privado.

Los refranes presentados fueron:

1. Para el grupo 1: "El que tiene buenos padrinos, no se cae dentro de la pila". "El que tiene padrinos, se bautiza”.

2. Para los grupos 2 y 3 agregamos los siguientes (siguiendo a Zalpa, et. al., 2014): "Es mejor tener amigos que dinero". "El que al buen árbol se arrima, buena sombra le cobija".

Los discursos fueron registrados en video y se transcribieron para un procedimiento de codificación y recodificación, en el que inductivamente fueron identificándose como categorías los repertorios interpretativos.

\section{Resultados}

Aunque no todos los participantes conocían todos los refranes, asociaron pronto su sentido con las ventajas que tienen los vínculos de amistad para la ayuda y la protección. Sin embargo, estos recursos no se ven siempre como algo positivo, pues se considera que podrían acostumbrar al facilismo y además confieren cuestionables ventajas sobre los demás. La doble evaluación que emerge sobre el uso de padrinos, señalando de una parte sus efectos negativos en la formación del carácter personal y cuestionando, de otra, sus implicaciones desde consideraciones de imparcialidad, establece la base de la comprensión moral de la corrupción a lo largo de todas las discusiones.

Identificamos tres repertorios interpretativos que denominamos de la siguiente manera: 1) de la victimización; 2) de la aceptación-justificación; 3) de la superación ética. Aunque cada uno de estos repertorios se despliega en torno a sus propias figuras, encontramos que los tres repertorios están atravesados por la metáfora central del movimiento (impedido, limitado y conquistado).

El primer repertorio, el de la victimización, emerge en el paso de la comprensión del intercambio de favores como forma de reciprocidad comunitaria, al señalamiento del uso que otros hacen de sus influencias de una manera éticamente cuestionable, para lograr un trato preferencial en la imposición de la ley o en la asignación de recursos y cargos:

Grupo 1, estudiante 3 (G1E3): el refrán lo puedo asociar con la experiencia que tuve la semana pasada. Un grupo, así como estamos aquí, discutiendo sobre qué apoyo se le está dando a los jóvenes, cómo se está ayudando a los jóvenes para que salgan al progreso. Muchos decían: "no, es que los jóvenes no quieren"; pero, en realidad, lo que pasa es que... Yo vivo en un pueblo pequeño y al vivir en un pueblo pequeño todo se queda ahí entre pocas personas. Los pocos recursos que llegan se quedan entre grupos y muchos jóvenes con talento, con índice alto, no pueden alcanzar sus deseos de estudiar. Por ejemplo, ir a la universidad ${ }^{* *}$ con una beca, que allá se ofertan muchas becas para la universidad ${ }^{* *}$, pero solamente se las dan a las personas porque tú eres hijo del síndico, tú eres primo del síndico, tú eres primo del que dirige el Distrito, eres hijo de fulano eres el amigo de perencejo y las noticias se quedan entre ellos.

Yo tiendo a escuchar a las personas cuando van a hablar y me dicen: "no, yo quería ese puesto, yo estaba luchando por ese puesto, llevé mis papeles y cuando cumplí, viene fulano, que ni siquiera se ha esforzado." Incluso personas que a veces no han terminado la carrera docente ya tienen un nombramiento, antes que otra persona que ha terminado y tiene tiempo esperando y yendo a licencias no logra conseguir. Hay que tener a alguien que te empuje para poder llegar, alguien que te represente. Ya tu talento, tu nivel académico no vale para nada, lo están poniendo por el suelo, le están diciendo a los jóvenes: bueno, no estudies, no hagas nada, tú solamente consíguete a alguien que te ayude, que te impulse, y eso no es un buen ejemplo. 
G1E5: realmente esa frase alude mucho a lo que está pasando hoy en día en la República Dominicana, por ejemplo, con los maestros. Si usted no tiene una buena cuña, no tiene un buen padrino, o no tiene una buena relación con los políticos, usted no consigue lo que es el nombramiento. El otro día se me acercó un joven, un compañero mío que es un pastor allá, muy buenos amigos somos. Él está haciendo campaña para ${ }^{* *}$, y me estaba diciendo que tenemos que apoyar a xx y yo, realmente como que, o sea... no me llamó la atención eso y él me trató de convencer y me decía: "tú tienes que tener en cuenta que hoy en día la fuerza para todo es la política, si tú necesitas un buen empleo por medio de la política se te puede facilitar, tú mayormente lo tienes que aceptar". Le dije yo: ¿pero por qué? "Porque tú estás estudiando educación.” ¿Pero qué tiene que ver eso? "No, sabes que ahora mismo con esa buena cuña que tu tengas te puede facilitar el asunto"; o sea que ya hasta la educación, al maestro, ya esto se está manipulando a través de cuña, ya no vale su conocimiento.

La metáfora del movimiento impedido o limitado emerge con claridad al tratar de explicarse el significado del padrinazgo recurriendo a las figuras de "la palanca", "el empujón”, "el impulso". Pero se prolonga en el sentido del "sostén" que no deja caer, "el estar pegado" (a lo que se mueve, porque no es posible el movimiento autónomo). En estas significaciones, la metáfora permite la conmutación al segundo repertorio interpretativo de la corrupción, el de la aceptación-justificación, que fue el que ocupó la mayor parte de las discusiones. La resuelta acusación de las influencias como generadoras de inequidad, se desplaza a un discurso indulgente en el que se justifica su uso por necesidad, por las circunstancias o porque se poseen las capacidades y solo falta un pequeño empujón para que prosperen:

G1E7: también se le dice "palanca". Aquí no se menciona mucho ese término, pero cuando estuve por allá se decía eso de palanca, que es algo que te impulsa porque no es que, o sea, se llega de las dos maneras, pero si uno no está con esa palanca da más brega que yendo por la ley y mucha gente cuando ve difícil irse por la ley, por el camino del bien, cuando lo ven complicado, se van para la palanca porque los catapulta.

Moderador: ¿tener palanca es importante?

G1E7: a ver, es importante pero no es lo correcto, por el hecho de que, mire: cuando alguien está en una necesidad y quiere progresar, tanto por él como por su familia. Él, bueno, hace todo lo posible por lo correcto, envía currículo, pasa trabajo, a veces llega tarde a su casa, muchas veces con malas impresiones que se ha llevado de sus superiores cuando va al puesto de trabajo. Pero resulta que hay un amigo o un sujeto que lo puede ayudar en eso. El joven tranquilo se queda mientras que el otro le va haciendo el arreglo y lo que le tomó mucho tiempo y no lo consiguió, lo consiguió en base al amigo. No es lo correcto. porque o.k., pero ya era necesario porque es así, tenía que vivir, él debía superarse.

G2E3: bueno, yo necesitándolo, ¡porque ahí es que vamos!, porque es que también es la necesidad, porque ahí iba a agregar a lo que dijo E8 [refiriéndose a la anécdota sobre alguien que no escogió a un pariente para un trabajo, F.G.], que a sus familiares ella los eliminó, porque hay también que pensar: no, pero es que ella lo necesita, por ejemplo, es una persona que tiene tres niños pequeños, que es madre soltera, o sea, no tiene cómo mantenerse. Ya ahí sería diferente, o sea, estamos hablando de una necesidad.

Moderador: ¿y si todos lo necesitan?

G2E3: porque es que... o sea.. también eso es cierto ahí... pero es que volvemos al tema de que no es como debe de ser, sino como es la realidad.

Lo que "es", la "realidad", remite a otro posicionamiento, a otras lógicas de acción orientadas por normas que si bien no niegan las valoraciones iniciales, las dejan en suspenso. Con ello se acata en la práctica por un tiempo indeterminado lo que impone el actuar colectivo, si bien se deja intermitentemente constancia de que se hace contra la propia voluntad y convicción: 
G1E6: la pregunta es que si es importante. Importante sí, por el contexto. O sea, ya estamos obligados a tenerlo no porque sea correcto. Como dice E7, no es correcto porque se supone que estamos siendo formados con ciertos valores éticos y morales donde te enseñan a que tú tienes que alcanzar tus logros por tus propios medios, que la educación que te están dando tiene que sacarte a camino, ¿no? Porque ese es el fin de la educación: que tú puedas alcanzar lo que tú quieras a través de tus propios méritos y eso te llena de satisfacción más que tú tener una propia cuña; ah, no, que yo conozco al hijo de fulano y que fulano le va a decir a su papá y su papá le va a decir a su mamá y su mamá me va a pegar con fulano. Entonces, es importante debido al contexto y a la sociedad en la que estamos metidos. Pero lo correcto sería que tú puedas llegar con tus propios méritos adonde tú quieras llegar.

G2E6: Pero es una realidad lo que él dice en cierto caso [refiriéndose a E8, quien ha afirmado que si se es realmente bueno no se necesitan cuñas, F.G.] Pero hay que ver el sistema en el cual uno vive, un sistema un tanto corrupto, donde no siempre se fijan en la capacidad intelectual de la persona, sino en el apego emocional, ya cualquier moderador, no simplemente en el ámbito barrial, sino en cualquier conferencia, cualquier moderador te dice, aunque en ciertos casos te lo rodea, en ciertos casos te habla que es bueno buscar a alguien que sea tu sustento, no te dice un cuña en sí pero ellos mismos entienden que aquí no, desde la óptica de tú mismo, no se fijan en la capacidad, sino en el que está pegado dicha persona, no se mira tanto lo intelectual.

En la "realidad", el conjunto de capacidades y habilidades que deberían contar como criterio determinante para la asignación de recursos y oportunidades, se doblega ante el peso de las relaciones personales, del capital social de padrinos y "cuñas" que se posea. Sin embargo, en este repertorio el mérito no se reivindica como punto de apoyo para oponerse decisivamente a la lógica de los padrinazgos, sino que, por el contrario, la creencia individual de que se posee dicho mérito se moviliza como justificación para hacer uso de padrinos. De esta manera, se resuelve el eventual conflicto moral que podría surgir al buscar un "empujón":

G1E4: es importante tener una palanca en el contexto de lo que se está viviendo hoy en día porque si tú no la tienes no vas a llegar a nada, o sea, tendrías que esperar diez, quince años para lograr algo que tus capacidades, según tus capacidades podrías lograr en uno o dos o tres, pero por la situación que tú vives entonces se te dificulta.

G1E5: ahora E4 dirá lo mismo: yo tengo el conocimiento yo sé que puedo dar mucho de mí, yo sé que puedo levantar una sociedad diferente; E7 dirá lo mismo. Ellos esperan poder hacerlo legalmente, o sea, entrada normal, porque yo siempre he dicho: tampoco vamos a tapar el sol con un dedo; nosotros queremos una sociedad diferente, pero si ustedes saben, tienen la capacidad y tiene la cuña, igloria a Dios!

G2E7: y tampoco podemos verlo como todo negativo, porque si yo tengo un cuña y soy muy buena no tendré que pasar por una entrevista; simplemente, él me va a facilitar ir directamente al área en la que yo me voy a ejercer en el trabajo, si soy buena, me haría ese grande favor.

G3E4: seguir a los funcionarios que están en el poder nos va a garantizar en un futuro un trabajo estable ¿qué dirías si nosotros no lo seguimos? Quizás no vamos a conseguir el trabajo que deseamos, como lo decimos, por la lucha que tomamos, que nos preparamos como profesionales y lo va a conseguir otro que, como dice mi compañero, [tiene] la cuña, aunque no está preparado. Entonces no lo utilizamos porque no lo tenemos. Ahora, si lo llegáramos a tener, te aseguro que sí lo utilizaríamos.

G3E8: yo lo haría. Además, si siento que estoy preparada, mucho más rápido.

En este repertorio de la aceptación-justificación, el "cuña" aparece también como un recurso de protección ante el abuso y la injusticia de que se es víctima, como refleja el siguiente fragmento del segundo grupo focal, en el que se aprecia tanto la variabilidad de repertorios en un mismo participante (E8), como la 
transitoria desorientación provocada por el retorno de la conversación al primer repertorio:

G2E8: bueno eso es trampa, eso depende de la situación en la que la persona se encuentre porque, por ejemplo, sucede que aquí en nuestro país tenemos muchos problemas ya que, con la policía, que hacen su trabajo bien, otras veces lo hacen mal, otras veces cometen abusos. Por ejemplo, supongamos que un policía hoy comete abusos con usted y lo mete preso sin el policía tener razón, sin usted haber hecho nada, sin usted tener la culpa; sucede que usted tiene una cuńa que, digamos, tiene algún cargo en el ejército, supongamos que es coronel, teniente o tiene algún tipo de rango, entonces usted tiene su teléfono, llama a su cuña, "hola, me pasó tal y tal cosa, fulano de tal y fulano de tal, me metieron preso sin yo hacerles nada”, etc. ¿Qué va a suceder? Pues su cuña dará la orden de que lo suelten, entonces ese es uno de los casos en que podemos utilizar cuńas.

G2E4: ;que son buenos!

G2E6: depende del área.

G2E7: por esa misma razón, a pesar de que usted haga algo y tenga una buena cuña, como decimos, esa persona va a ignorar la malicia que usted realizó para, de alguna manera, sacarlo y librarlo de todo lo que usted hizo. Esa es una de las desventajas que aquí se realiza mucho.

G2E8: he visto muchos casos donde personas cometen actos en contra de la sociedad y, por ejemplo, al mismo día o al otro día son liberados, o sea, sin tener razón, simplemente por tener algunos familiares que ocupan puestos, hablan con ellos: "deja salir a fulano que ese es primo mío, familia mía, hermano mío”, y simplemente lo hacen.

G2E7: también se hace mediante un buen soborno.

Moderador: ¿entonces sí se justifica usar cuñas? G2E5: si el caso lo amerita.

G2E4: no necesariamente tiene que ser algo malo, puede ser un trabajo.

Moderador: y si para el trabajo hay, como en el caso que ustedes mencionaron, cinco personas y yo me presento y quiero que me den el trabajo, pero los otros son mejores que yo, ¿eso importaría?

G2E8: sí, sí importa.

G2E6: ¡no! Yo entiendo, como le digo, si otros lo son más, pero estamos hablando del cuña; si el cuña es el líder o ya sea el gerente de la empresa, aunque los otros sean un poco más competentes que yo, yo creo que a mí me darían el trabajo porque mi cuña es el gerente no el de ellos y eso influye mucho y se ve en el diario vivir.

G2E8: bueno, yo opino lo contrario. Si de ser tu cuña te darían el trabajo aunque los otros fueran más competentes, es lógico, es lo que siempre sucede, pero yo opino que eso está mal porque si, por ejemplo, nos ponemos a pensar: hay cinco personas, esas cinco se prepararon muy bien, supongamos que duraron cuatro años de carrera preparándose bien para ser buenos profesionales, tienen maestrías, posgrados, otros cursos, están muy bien preparados. Yo no he hecho nada de eso, no he trabajado en nada de eso, no sé lo que es la universidad, pero tengo un cuña, entonces yo voy por el puesto, ¿qué opinan ahí? ¿Es correcto que me lo den a mí o a ustedes? ¡Pues claro que no! Es correcto que se elija al más competente. Yo opino que las cosas se deben hacer por las reglas, ¿qué no se hagan así? Bueno, pues sabemos que realmente no se hacen así y aunque haya muchos países donde lo hagan así, en ningún país se hace del todo correctamente.

G2E6: creo que no entendí, sería que usted preguntó, ¿lo correcto o lo que se hace?

Otro elemento a apreciar en este repertorio de la aceptación-justificación es el de las obligaciones que impone el tejido de vínculos personales y comunitarios a quien está en posición de otorgar, expectativas de comportamiento cuyo incumplimiento conlleva sanciones microsociales y emocionales. La lealtad se antepone al mérito y la lógica del intercambio privado de favores se moviliza hacia lo público. La evaluación de las capacidades se posterga por la indulgencia que se espera del "cuña" ante la posibilidad de aprender: 
G2E5: por ejemplo, en el caso del cuña, si yo tengo un pariente, yo estoy en una empresa a cargo o soy gerente y tengo un pariente que está capacitado para tener ese puesto, pero hay otra persona que está mucho más capacitada que mi pariente, ¿qué pasa? Que el cuña siempre va a elegir su pariente porque, ¿qué es lo que pasa? Si no lo elige ese pariente se va a ir a lo que es el ambiente personal o emocional.

G2E1: y la familia también.

G2E5: claro, entonces va a decir: "bueno, pero yo soy tu familia o yo soy tu amigo, ¿por qué tú no me elegiste a mí si tú eres el gerente?” Entonces eso va a crear una enemistad. El cuña, por temor a que esa amistad se rompa entre ellos, siempre va a elegir a la persona más cercana él.

G2E5: entonces si tenemos un aliado, o conocemos algún director, debemos hablar con él y explicarle y él lo va a facilitar porque es así, aunque se vea mal siempre será así, porque cada persona siempre ayuda a las personas más cercanas a él; por ejemplo, si yo tengo un vaso con agua y ellos tienen sed yo se lo daría a la persona con quien más yo comparto [...] Aunque esté mal siempre será así, porque las personas siempre nos vamos a lo que es el ámbito emocional, o sea, que me sentiría mal si no se la doy primero a mi amigo o conocido.

G2E1: yo digo que hay que actuar de la manera correcta, pero uno entendiendo que si yo sé de un trabajo en el cual está mi cuña y yo le digo: yo tengo una buena capacidad de adquirir conocimientos, y él me dice: "bueno, te vamos a dar el trabajo, pero vamos a ver qué pasa”, ya ahí uno consigue trabajo y si yo no doy para eso, el cuńa va a estar consciente de que yo no puedo estar ahí, entonces tomará la segunda medida que será sacarme del trabajo.

Sin embargo, en comparación con la necesidad y con la creencia de que se tienen los méritos, la justificación por lealtad y benevolencia alcanza un consenso más débil, por las implicaciones que tiene, si se consideran los requerimientos y responsabilidades de los trabajos profesionales, y en este caso, los que conlleva el ejercicio de la docencia:
G2E8: bueno, esa es la postura general, pero también existen sus excepciones porque en algunos casos hay esos que son muy firmes. Por ejemplo, yo tengo una amiga que trabaja en una empresa por ahí por la zona y fueron varios de sus familiares que necesitaban obtener trabajo y al ellos no estar calificados ni reunir los requisitos, pues ella simplemente los eliminó [...] El profesor ${ }^{* * *}$, la mujer de él estuvo tomando clase con él, ella no pasó con los contenidos, ¿y que le sucedió? Él la quemó [reprobó. F.G.]. Entonces muchas personas, muchos estudiantes decían: "yo lo mato, yo le hago esto". ¿Pero qué sucede? Que él hizo lo correcto.

G2E3: pero ella se dará cuenta en algún momento que será la beneficiada, porque es que también por eso es que muchas veces hay personas con títulos en nuestro país y yo sé que no solamente aquí, también hay otros países que pasa lo mismo, $\mathrm{y}$ es que hay personas profesionales mediocres, $\mathrm{y}$ es porque se trata solamente de adquirir el título, pero no el conocimiento.

Las tensiones que asoman en este punto sitúan de nuevo las discusiones en el terreno de "lo que deber ser”, pero con un elemento adicional: la consideración de lo que efectivamente tendría que hacerse en primera persona para contrarrestar prácticas comunes que se ponderan reprobables si se evalúan tanto desde principios universales como desde las consecuencias que acarrean socialmente en el mediano y largo plazo. Con este desplazamiento arribamos al tercer repertorio interpretativo identificado: el de la superación ética de la corrupción. Las consideraciones que aquí emergen permiten apreciar dos posturas, dependiendo de si se hace énfasis en una noción impersonal de equidad o se resalta la idea del cultivo de una virtud personal basada en la perseverancia y la abnegación para "hacer la diferencia" a través del ejemplo.

Recordemos el pasaje mencionado de la discusión en el segundo grupo, cuando el participante E6, ante el cuestionamiento de sus compañeros por validar el uso de padrinos, expresaba su vacilación sobre cuál era propiamente el marco en el que se estaba discutiendo, el del ser o el del deber ser: 
G2E6: creo que no entendí. ¿Usted preguntó, por lo correcto o lo que se hace?

Moderador: tú dices "es mi cuña y yo recibí el trabajo”. ¿Y si te presentas, y consideras que eres más competente que los demás, pero hay otro ahí que tiene un cuña, tú lo aceptas porque es lo usual?

G2E6: ¡exacto! Porque le fui sincero, yo sé que si, por ejemplo, yo conozco, aunque yo entienda que soy un poquito más competente, estoy un poco disgustado a sinceridad, pero reconozco que quien tiene cuña es al que le dan el trabajo. G2E4: yo armo la real guerra así.

G2E6: no, porque al final la gana él, porque él es que tiene el cuña.

Moderador: ¿una guerra?

G2E4: ¡una guerra!, porque no es justo.

Este desplazamiento implica la idea de que lo justo, la consideración imparcial de los intereses, debe ser lo que se imponga a los hechos y no que lo fáctico determine lo que se ha de aceptar como correcto. La misma oposición se manifiesta en el primer grupo, ante la postura expresada de que la necesidad y el uso generalizado de los padrinos convierten esta práctica en "obligación" y que los inconvenientes éticos se dispensan por la posesión de capacidades. Allí, la situación hipotética en discusión pasa a definirse a la luz de la responsabilidad subjetiva y de la agencia individual consciente para romper con prácticas de corrupción:

G1E3: si vemos nuestra sociedad actualmente se puede decir que para conseguir un empleo se hace necesario tener una cuña, o una palanca, o un padrino, pero no es lo correcto. Inmediatamente nosotros recurrimos a un padrino, pues estamos entrando a toda la corrupción, nos estamos corrompiendo, digámoslo así, porque no hay mejor satisfacción que lograr algo por nuestro esfuerzo. Por ejemplo, tenemos todo este tiempo aquí, esforzándonos, tratando de salir a camino con una licenciatura, para que cuando salgamos allí afuera, a la sociedad, no sea por nuestros méritos que tengamos esa premiación, sino que haya sido sencillamente por tener una cuńa.
Si el saber que se cuenta con padrinos hace innecesario el esfuerzo que contribuye a la formación del carácter, lograr las cosas por el laborioso camino correcto es algo que produce un sentimiento de satisfacción y una noción más definida de la propia valía, además de poseer un valor ejemplar para "hacer la diferencia”. El aprendizaje de una paciente espera a que "llegue el momento", se expresa en la idea del respeto del lugar en la fila. Sin embargo, su sentido no siempre es claro en un contexto de débiles sanciones sociales en el que la solitaria acción individual parece producir más bien héroes trágicos:

G1E2: yo digo que en el país, así como lo estamos llevando ahora mismo, es más que bueno, necesario. Poner este pequeño ejemplo: antes decir... no podemos decir yo voy a hacer la diferencia ¿por qué razón? Con este ejemplo les explico por qué no decir "yo voy a hacer la diferencia". Mis queridos compañeros: todos lo hacen cuando vamos a comer [se refiere al comedor del Recinto, F.G.] quien llega de último quiere ponerse adelante y todos van pasando de una y yo digo: yo voy a hacer la diferencia, yo me voy a quedar en el lugar donde estoy, pero veo que pasan cinco, se ponen adelante, pasan diez, se ponen adelante, pasan veinte y se ponen adelante y yo soy la diferencia y me quedo mirando mientras todos pasan. Es decir, yo soy la diferencia, yo soy el que me estoy esforzando para llegar a un lugar mientras veo que aquellos que no se esfuerzan porque tienen esa cuña pasan como si nada y me dejan, mientras que estoy tratando de hacer la diferencia en el mismo lugar. Eso como que lo hace a uno sentir impotente y dice ah, yo también necesito tener la cuña yo tengo que tener esa cuña si quiero llegar a hacer algo.

G1E6: chistoso que él dice: "ah, me quedo mirando y ya, todos pasan". Pero me ha sucedido que muchas veces yo he dicho: no, en $\mathrm{X}$ cosa yo voy a hacer la diferencia y yo digo cónchale, o sea, cualquiera... deja de ser la diferencia. Sí, ¿pero sabes qué pasa? Siempre hay alguien que te está mirando, siempre, o sea siempre alguien nota que quieres ser la diferencia. Algún día 
alguien va a decir: “¡Pero X nunca se pasa, no se le pasen a ese muchacho!' Y tú te vas a dar como que... alguien se dio cuenta y nadie se te va a pasar. Ok, tal vez van a pasar 20 ańos (risas) pero va a suceder, va a suceder porque yo creo que la sociedad es como ret roactiva: lo que pasa quizá en el siglo i vuelve y pasa en el xx, y lo que pasa en el xx vuelve y pasa en el XL, yo creo en eso. Entonces algún día esto que tú estás haciendo se te va a devolver.

G1E1: y hay algo que a mí me gusta decir: si yo hago las cosas de la manera correcta, aunque no me salga a la primera, aunque no me salga a la segunda, allá arriba hay un Dios que todo lo ve y va a llegar el momento en que Él, que es un Dios justo, va a traer justicia y va a permitir que lo que yo estoy esforzándome para conseguir, si es para mí, Él me lo va a dar.

G1E4: yo quería decir algo acerca de lo que E5 dijo. Es cierto: hay muchos maestros que están mucho más preparados que otros y no tienen la oportunidad porque no tienen una cuña. Sin embargo, aunque tú seas un maestro preparado y tienes la cuña, si la utilizas no te estás yendo por el camino correcto, estás errando. Entonces Dios te está mirando y Él no va a decir: "ah, no, él sí tiene la oportunidad y yo se lo voy a perdonar”. No, Él va a notar eso también.

La consideración crítica de las consecuencias de la propia acción supone el reconocimiento de un orden moral que no pierde su significación por el hecho de que solo imperfectamente se refrende a través de hábitos sociales. A pesar de la pluralidad que recoge, la idea de un orden, sea determinado por principios universalistas, por la incipiente mirada de un otro generalizado o por la mirada enjuiciadora de Dios, constituye la especificidad de este tercer repertorio interpretativo de la superación ética de la corrupción. Asociada con ella está la expectativa de que las acciones individuales pueden tener un impacto transformador en los hábitos colectivos:

G1E7: uno está muy bien, perfecto, cumpliendo todo lo de la ley, el día que uno... porque es que la sociedad influye también, si todo el mundo se está yendo adelante y nadie dice nada pues adelántate tú también, porque eres parte del medio. Ah, pero te fuiste adelante y aparece uno, que eso está mal y entonces te hacen pasar una vergüenza ¿pero entonces por qué no le hicieron pasar vergüenza a esos que se entraron ahí?

G1E6: ese es el problema, que yo considero que somos extremistas, no solamente los dominicanos, yo creo que en todo el mundo: siempre acudimos a ver lo malo antes que lo bueno y de hecho hay alguien que siempre dice: “¡cónchale, pero pilas, este lo está haciendo bien!" Solo que es la minoría, ese es el problema, siempre es la minoría. Pero si así como quizás la minoría se levanta y dice: yo me voy a mantener en esta postura y la mayoría hace lo mismo, pues al fin y al cabo algo va a tener que pasar, o sea, se va a acabar esto de estar buscando cuñas.

La consideración abstracta de "otros", afectados en el mediano y largo plazo por las acciones puntuales de un agente que prioriza la satisfacción de sus necesidades inmediatas, permite identificar la emergencia de una representación de lo público como espacio abstracto en el que convergen complejamente consecuencias no intencionadas de las acciones individuales. Los efectos de tales acciones en los demás y en uno mismo se evalúan entonces desde la perspectiva de los miembros de una sociedad que reflexiona sobre el sentido de la formación profesional o el valor social de la confianza, más que desde la expectativa aislada de reconocimientos individuales:

G1E3: si esa persona no tiene los contenidos, no tiene una estrategia, los perjudicados en sí son los niños. Y esa persona contribuye a dañar la sociedad porque muchas veces esos niños llegan a básica, a media, llegan al bachillerato y muchas veces esos profesores que no tienen ese dominio cuando van al currículo y tienen que elaborar su planificación, muchas veces cuando no entienden el contenido lo que hacen es que lo vuelan y ahí el niño se pierde de conocer un tema. Ya cuando avanza el niño no tiene dominio de ese contenido y eso le va a perjudicar, lo va perjudicando a medida que va elaborando su proceso educativo. 
G1E6: sabes que no solamente denigra al niño sino que también, si tú acudes a la palanca en este caso y por ejemplo tú vuelas los contenidos, no estás preparado para ello, hace que ya la gente no confíe en los maestros [...] o sea, no solamente afectamos a un niño que tal vez es minoría, que es pequeño o que no tiene voto todavía y tal vez ni siquiera voz, sino que nos hacemos daño a nosotros mismos.

G1E4: la cuńa afecta todo eso, pero afecta al estudiante que algún día va a integrarse de manera definitiva a la sociedad. Uno, afecta al sistema en sí porque no vamos a darle clase a un niño, sino a varios que van a formar parte del sistema. Dos, me afecta a mí misma como maestra y como persona porque estoy perdiendo mis valores, mi moralidad, estoy dudando de mi capacidad como maestra para adquirir un puesto. Y tres, altera el bienestar de la familia porque un niño que no tenga un desarrollo escolar óptimo, no todo es trabajo de la escuela, pero si en la escuela no te enseñan lo necesario, ¿cómo tú vas a sostener un hogar? O sea, creo que afecta más que nosotros busquemos una cuña. Si no me están nombrando en Educación me voy a buscar otro empleo, tu familia no se va a morir de hambre. Puede ser que tú en vez de cobrar 35 o 45 mil pesos termines cobrando 15 mil, pero al menos tu familia come todos los días, al menos tienes un trabajo honrado, al menos no estas dañando a nadie con tu trabajo, no sé. Digo; hay muchas formas. Yo pienso que tomando la cuña no hay justificación; luego de que existieron las excusas nadie queda mal.

G2E8: un cuña en un cargo público con sus decisiones o sus malas decisiones podría afectar prácticamente todo el país, porque las cosas públicas son de todos. Por ejemplo, en el caso que mencionábamos anteriormente del profesor de inglés, si usted es el cuña de él y él no está capacitado pero usted tiene gran autoridad, entonces déjame ponerlo a él porque es mi familia o lo que sea, ¿qué sucederá? Que a los alumnos que trabajen con él no les va a poder enseñar, no van a tener una correcta educación, ¿y quiénes serán ellos? Pues el futuro del país, entonces nos afectará a todos.

G1E1: por el error del médico se muere; por el error del abogado se queda preso; pero el error de un maestro se multiplica.

Al final de las discusiones lanzamos en los grupos la pregunta por su idea de lo público. En el grupo dos, el de los estudiantes recién ingresados, las respuestas se relacionaron predominantemente con estructuras gubernamentales y administrativas: "todo lo perteneciente al Estado" (G2E6); los servicios que para su funcionamiento "dependen de los impuestos que pagamos todos" (G2E1); "las instituciones o servicios que no son privados." (G2E2); "aquello que nos pertenece a todos" (G2E8). En el tercer grupo, del sexto cuatrimestre, lo público se asoció con "el Estado, para todos" (G3E2); "lo que involucra la sociedad" (G3E5); "lo público son los otros" (G3E6). La cercanía con lo público está mediada por la experiencia de ser estudiante del instituto: "realmente, yo ahora mismo siento que es cercano porque estoy estudiando en una entidad pública (G3E3). En cuanto al primer grupo, el de los estudiantes más avanzados, donde se tematizó especialmente la idea de un espacio difuso en el que se desatan consecuencias no intencionadas de la acción individual, resaltamos una expresión: lo público es "una reacción en cadena” (G1E7).

\section{Discusión y conclusiones}

Los pasajes citados ilustran los repertorios interpretativos de la victimización, de la aceptación-justificación y de la superación ética, así como las metáforas de movimiento en los modos de describir, condenar o justificar la corrupción: el movimiento impedido, que la significa como obstáculo y limitación; el movimiento apoyado (palanca y catapulta), que la muestra como un recurso necesario; y el movimiento autónomo, conquistado por virtudes y convicciones fuertes. La aparición de estos repertorios no se da necesariamente de manera secuencial, sino que sus discursos se despliegan con frecuencia de manera paralela, generando puntos de tensión que dan la impresión de retornos a posiciones que parecían ya desechadas. 
La recurrencia entre el rechazo y la justificación de la corrupción permite apreciar una variación no solo entre diferentes participantes, sino a veces también en un mismo sujeto.

De acuerdo con el primer repertorio, la corrupción se urde en otros lugares, donde están quienes detentan poder, y la padecen quienes están sometidos a él. La corrupción deteriora el acceso equitativo a las oportunidades, al imponer de facto una asignación de recursos y posiciones basada en las relaciones personales antes que en el mérito. Sin embargo, el mérito funciona como argumento, tanto para el rechazo de esas relaciones en la forma del amiguismo que arrebata oportunidades, como para su justificación, porque el contar con un padrino o una "cuña" le permite al beneficiario aspirar a lo que cree que merecería, pero se le niega. Conforme se avanza en el segundo repertorio, esta idea de la compensación basada en las capacidades personales o profesionales, deja de ser central y se combina con demandas y expectativas de favorecimiento basadas en la pura lealtad del vínculo, para finalmente dejarse completamente de lado el mérito y apelar a la indulgencia del dador, incluso ante una clara ausencia de méritos.

En el centro del tercer repertorio está la idea de que es posible modificar prácticas de corrupción socialmente aceptadas, a través de cambios en la persona, aunque la persistencia de aquellas produzca con frecuencia la sensación de que la acción individual es un gesto inútil, sin impacto en el entorno. En ausencia de sanciones sociales claras, la instancia de cambio es el sujeto que conscientemente ajusta su comportamiento moralmente, bien sea porque cultiva su autoimagen como persona virtuosa, porque cree que existe un equilibro cósmico de acciones y reacciones, porque teme a la mirada vigilante de Dios, o porque en él toma forma la imagen de un otro abstracto, posible afectado por las propias acciones, que se convierte en anclaje de una noción de lo público.

Nuestros hallazgos concuerdan con lo observado en otros estudios, en cuanto a que prácticas de corrupción se confunden con las lógicas del intercambio de favores y de allí parasitan de las normas tácitas de lealtad y protección que rigen relaciones privadas de amistad y parentesco (Graycar y Jancsics, 2016). A pesar de hallar un repertorio de justificación de la corrupción, al discutir sobre su presencia en situaciones cercanas a las propias vivencias de los participantes, no se manifiesta una reivindicación abierta de ella como "un acto de rebeldía contra un sistema legal que legitima una estructura social desigual" (Gil-Villa, 2013, p. 272). Por el contrario, y a pesar de cierto reconocimiento de las situaciones de necesidad, las justificaciones corren con la carga de la prueba frente a una evaluación predominantemente negativa.

También encontramos elementos de una lógica de la acción colectiva, como la que han señalado Rothstein y Torsello (2013): abstenerse de hacer algo que los demás hacen (posicionarse adelante en una fila mediante el uso de influencias), trae desventajas para el individuo que, aunque los desapruebe y sin que esté motivado por una "codicia" particular, se ve empujado, en condiciones de carencia, a adaptar su conducta a esquemas de respuesta aceptados socialmente (Ntayi, Ngoboka, Kakooza, 2013), no necesariamente para obtener una mejor posición, sino para conservar la posición inicial.

Huber (2008) ha afirmado que, en razón de la dificultad para acceder a la práctica real de la corrupción, que lleva al investigador a depender del discurso con el que los sujetos la describen y evalúan, los estudios empíricos sobre corrupción son, en realidad, estudios del desarrollo del juicio moral. Esta observación resulta especialmente significativa en un estudio como el nuestro, enfocado en la manera como las personas hablan de corrupción. Pero la relación con el juicio moral no surge solo de la mirada externa del analista, pues encontramos que lo que desde la perspectiva analítica denominamos "variación", empieza a ser definido por los participantes, en el repertorio interpretativo de la "superación ética", en términos de inconsistencia moral, dado que, aun ante la ausencia o la debilidad de sanciones legales y sociales, cada individuo tendría, en principio, la posibilidad de elegir si se involucra o no, como dador o receptor, en cierto tipo de transacciones que por lo general considera incorrectas.

Esta apreciación formal deja al sujeto solo ante la disyuntiva de plegarse a reglas tácitas de supervivencia o mostrar una virtud ejemplar para asumir los altos 
costos que implica "hacer la diferencia". Es aquí donde adquieren especial relevancia las instituciones educativas, en cuanto tienen la posibilidad de integrar la responsabilidad moral subjetiva en un proyecto colectivo de ciudadanía que lleve la discusión sobre la corrupción, más allá de la abnegación individual y del debate interno de la propia conciencia, a un espacio intersubjetivo en el que se tematicen los implícitos que subyacen a las prácticas de corrupción y se normalicen sanciones sociales con una exigibilidad pública.

Esto implica una reflexión sobre la manera como se lleva a cabo la transición de la micro moral de las interacciones cotidianas a la macro moral de nuestra vida como ciudadanos, y sobre la importancia de cultivar un sentido de lo público que involucre no solo la noción de los bienes comunes, sino también la perspectiva de los efectos de las propias acciones sobre otros y el valor de los espacios de discusión sobre lo que nos afecta y lo que queremos ser. En los grupos focales encontramos indicios de una comprensión de lo público en el segundo sentido, pero menos en el primero y el tercero.

Igualmente, la reflexión sobre la transición de la lógica de las relaciones personales a la de los intereses de ciudadanía debe considerar los imaginarios sobre el acceso, la subsistencia y el digno desempeño en el campo profesional. En nuestro acercamiento notamos que la idea que han adquirido los estudiantes sobre cambios recientes en los mecanismos de evaluación y nombramiento de docentes, anima la expectativa de contar en adelante con un marco de regulaciones, dentro del cual sería más viable conciliar las necesidades individuales y la responsabilidad social de la profesión.

\section{Referencias}

Bloor, M., Frankland, J., Thomas, M. \& Robson, K. (2001). Focus Groups in Social Research. London, Inglaterra: Sage.

Duits, L. (2008). Multi-Girl-Culture. An Ethnography of Doing Identity. Amsterdam, Paises Bajos: Vossiuspers/ Amsterdam University Press.
Edley, N. (2001). Analysing masculinity: Interpretative repertoires, ideological dilemmas and subject positions. En: M. Wetherell, S. Taylor, \& S. J. Yates (Eds.), Discourse as Data: A Guide for Analysis (pp. 189-228). London, Reino Unido: Sage,.

Friedrich, C. (2002) Corruption Concepts in Historical Perspective. En: A. Heidenheimer, \& M. Johnston. (eds): Political Corruption. Concepts and Context (pp. 15-24). New Brunswick, EE. UU.: Transaction Publishers.

Gephart, M. (2012) Contested Meanings of Corruption: International and Local Narratives in the Case of Paraguay. German Institute of Global and Area Studies - GIGA. Working Papers, (191). Recuperado de: https://www.giga-hamburg.de/ $\mathrm{de} /$ publication/contested-meanings-of-corruption-international-and-local-narratives-in-the-case-of

Graycar, A. \& Jancsics, D. (2016). Gift Giving and Corruption. International Journal of Public Administration, vol. 40(12), pp.1013-1023.

Gil Villa, F. (2013). Discursos sobre corrupción en México. Revista Sociedad \& Equidad, (5), pp. 259-275.

Heidenheimer, A. \& Johnston, M. (eds. 2002) Introduction to Part I, Political Corruption. Concepts and Context (pp. 3-14.). New Brunswick, EE. UU.: Transaction Publishers.

Huber, L. (2008) Una interpretación antropológica de la corrupción. Lima, Perú: IEP, PROÉTICA.

Kajsiu, B. (2015). A Discourse Analysis of Corruption. Farham, Inglaterra: Ashgate.

Ntayi, J.; Ngoboka, P.; Kakooza, P. (2013). Moral Schemas and Corruption in Ugandan Public Procurement. Journal of Business Ethics, 112(3), pp. 417-436.

Nye, J. (2002). Corruption and Political Development. A Cost-Benefit Analysis. En: A. Heidenheimer \& M. Johnston (eds): Political Corruption. Concepts and Context (pp. 281-300). New Brunswick, EE. UU.: Transaction Publishers.

Rothstein, B. (2014). What is the Opposite of Corruption. Third World Quaterly. 35(5), pp. 737-752. 
Rothstein, B; Torsello, D. (2013) Is Corruption Understood Differently in Different Cultures? The Quality of Government Institute Working Paper Series, 5.

Seligson, M. (2002). The Impact of Corruption on Regime Legitimacy: A Comparative Study of Four Latin American Countries. The Journal of Politics, 64(2), pp. 408-433.

Stefes, Ch. (2007) Measuring, Conceptualizing, and Fighting Systemic Corruption: Evidence from Post-Soviet Countries. Perspectiven on Global Issues, 2(1).

Šumah, Š. (2018). Corruption, Causes and Consequences. En: V. Bobek (ed.) Trade and Global Market (pp. 63-79). London, Reino Unido: Intechopen.

Svensson, J. (2005). Eight Questions About Corruption. The Journal of Economic Perspectives, 19(3), pp. $19-42$.
Torsello, D. (2011). “The Ethnographic Study of Corruption: Research Themes in Political Anthropology". The Quality of Government Institute Working Paper Series, 2.

Transparencia Internacional (2018). Índice de percepción de la corrupción. Recuperado de: https:// www.transparency.org/files/content/pages/ CPI_2018_Executive_summary_web_ES.pdf

Wetherell, M.; Potter, J. (1996). El análisis del discurso y la identificación de los repertorios interpretativos. En: A.J. Gordo y J.L. Linaza (edit.) Psicologías, discursos y poder. Espańa: Visor.

Zalpa, G.; Tapia, E., Reyes, J. (2014). “El que a buen árbol se arrima..." Intercambio de favores y corrupción. Cultura y representaciones sociales, 9(17), pp. 149-176. 